\title{
Découverte de solution et flexibilité cognitive
}

\section{Evelyne Clément}

CY Cergy Paris Université - Laboratoire PARAGRAPHE

\subsection{Introduction}

Dans la vie quotidienne, scolaire ou professionnelle, s'adapter face à un environnement changeant et ajuster son comportement en réponse aux évènements nouveaux ou inattendus sollicitent la flexibilité cognitive. En outre, développer de nouvelles compétences et connaissances, ou bien transférer et adapter des connaissances antérieures qui répondent aux contraintes de la nouvelle situation témoignent aussi de flexibilité. Enfin, plus spécifiquement, en situation de résolution de problèmes, découvrir la solution nécessite la plupart du temps de changer de point de vue, changer de représentation sur la situation et ainsi faire preuve de flexibilité représentationnelle ou conceptuelle.

Dans ce chapitre, après avoir présenté dans une première section ce que l'on définit comme une situation problème, évoqué le rôle des connaissances sur le monde et celui des processus de transfert impliqués dans la découverte de solution, dans une seconde section, nous situerons le concept de flexibilité plus particulièrement dans les recherches menées en éducation et en psychologie cognitive. Dans une troisième section, nous présenterons les résultats de travaux récents menés sur les liens entre flexibilité, créativité et performances académiques. 
Nous discuterons de l'intérêt tant d'un point de vue fondamental que des implications dans le domaine scolaire des recherches dont l'objet est le développement de programmes de stimulation de la flexibilité et de la pensée créative.

\subsection{Découverte de solution et flexibilité cognitive}

\subsubsection{Qu'est qu'un problème?}

Avant toute chose, une question est celle de savoir ce que l'on définit comme une situation problématique, ou en d'autres termes, ce qui dans le champ de la psychologie cognitive ou de l'éducation est défini comme un problème. De façon générale, cette notion recouvre une grande variété de situations dans lesquelles celui ou celle qui a l'intention d'atteindre un objectif, un but, ne connait pas la manière d'atteindre cet objectif. Une situation est ainsi définie comme un problème quand elle est nouvelle, non routinière, c'est-à-dire que les connaissances acquises dans des situations antérieures ne peuvent être appliquées directement dans la nouvelle situation. De la sorte, certains auteurs dans le domaine de l'éducation des mathématiques différencient les problèmes dits routiniers pour lesquels les stratégies et procédures apprises dans des exercices précédents s'appliquent dans la nouvelle situation, des problèmes dits non routiniers pour lesquels la solution requiert une pensée flexible et créative afin de trouver une façon de la découvrir (e.g., Elia et al. 2009). Ce ne sont pas tant les propriétés objectives d'une situation qui la définissent comme un problème, mais bien les connaissances et le niveau d'expertise que l'on a sur la situation (Krems 1995 ; Novick 1988). Dans la suite du texte nous qualifions problème toute situation relevant de problèmes non routiniers définis comme précédemment. En ce sens, nous considérerons que toute situation de résolution de problèmes est une situation d'apprentissage. Inversement, toute situation d'apprentissage est par nature une situation de résolution de problèmes dans laquelle nos connaissances antérieures vont être mobilisées mais sur lesquelles il faudra opérer des transformations, et faire ainsi preuve de flexibilité cognitive, pour maîtriser les concepts impliqués dans la nouvelle situation.

Un des mécanismes fondamentaux des situations d'apprentissage en général, et de la résolution de problèmes en particulier, est que face à une nouvelle situation, on cherchera à appliquer ce que l'on a appris dans des situations rencontrées antérieurement. C'est sur la base du connu que l'on tente d'appréhender le nouveau. Ce processus de généralisation est un des piliers de l'apprentissage, mais il peut arriver que les connaissances antérieures rendent difficiles la découverte de solution. Dans ce cas, il faudra changer de point de vue sur le problème et recoder la situation 
afin d'en sélectionner les propriétés non saillantes au premier abord, mais pertinentes pour atteindre le but fixé.

\subsubsection{Connaissances sur le monde, découverte de solution et transfert}

\subsubsection{Connaissances sur le monde et découverte de solution}

De nombreuses recherches montrent que l'expérience et les connaissances que l'on a sur le monde jouent un rôle majeur dans la découverte de solution d'un problème (Bassok et al. 1995). Par exemple, Clément et Richard (1997) ont montré comment les connaissances familières sur les actions qui permettent le changement d'état (changement de lieu ou changement de taille) pouvaient entraîner des différences très importantes de difficulté entre problèmes isomorphes qui partagent le même principe de solution mais dont les contextes évoquent des connaissances sur le changement d'état compatibles ou non avec la solution du problème. Pour tester le rôle des connaissances évoquées par les énoncés sur la plus ou moins grande difficulté des problèmes, les auteurs ont ainsi construit des versions pour lesquelles les connaissances sur les actions pour changer d'état permettent d'appliquer sans difficulté les règles d'action énoncées dans la consigne des problèmes, et d'autres où elles rendent plus difficile le respect de ces règles. Dans les problèmes de changement de taille pour lesquels le changement est conçu comme un processus continu de croissance, on observe chez les adultes des temps plus longs pour trouver la solution et/ou un nombre d'actions et de violations des règles plus élevé que dans les problèmes de changement de lieu où le changement est conçu comme un changement d'état compatible avec l'application des règles. Découvrir la solution dans les problèmes de changement de taille a été interprété par Clément (2009) comme l'expression flexible du changement de point de vue sur le problème.

En outre, le rôle des connaissances antérieures dans l'acquisition de nouvelles connaissances a été mis en évidence dans différents domaines et ceci tout au long de la vie (Vosniadou et Ortony 1989). Ce processus d'apprentissage par analogie est défini par certains auteurs comme étant au cœur de la pensée humaine (Hofstadter et Sander 2013). Dans le domaine scolaire, par exemple, le recours aux connaissances que les élèves possèdent sur le monde est un des moyens pour enseigner de nouvelles notions ou concepts. De la sorte, l'enseignement de nouvelles notions est souvent conçu sur la base de ces connaissances familières sur le monde que les élèves ont acquis par expérience. C'est ce qu'ont montré Bassok et al. (1998) en menant une analyse des manuels scolaires américains de mathématiques des grades 1 à 8 (qui correspondent en France aux classes de la grande section de maternelle à la classe de 5éme). Les auteurs rapportent que les relations sémantiques 
qu'entretiennent les objets qui sont présentés dans les énoncés de problèmes sont très fortement corrélés avec les opérations arithmétiques qui doivent être appliquées sur ces objets. Les auteurs notent que $97 \%$ des énoncés des problèmes d'addition, les objets à additionner sont des objets qui appartiennent à la même catégorie. Par exemple, il s'agira de billes rouges et de billes bleues, mais jamais des cookies et des paniers. De la même façon, $94 \%$ des problèmes qui requièrent une division impliquent des objets qui sont fonctionnellement reliés. Dans ce cas, les objets impliqués pourront être des cookies et des paniers, mais jamais des billes rouges et des billes bleues. De plus, quand on demande à de jeunes adultes, étudiants à l'université, de concevoir des énoncés de problèmes simples d'addition ou de division impliquant des paires d'objets- par exemple, des tulipes et des marguerites ou bien des tulipes et des vases- les étudiants ont tendance à construire des énoncés où la structure mathématique et la structure sémantique (c'est-à-dire les relations qu'entretiennent les objets), sont compatibles avec les paires impliquant des objets de la même catégorie. Quand il s'agit de tulipes et de marguerites, ils proposent des énoncés de problème d'addition. Avec les paires d'objets qui entretiennent une relation fonctionnelle (par exemple de contenance comme la paire tulipes et vases), ce sont des problèmes de division qui sont majoritairement proposés par les étudiants (Expérience 1, Bassok et al. 1998). Ce que révèlent ces résultats, c'est que les participants ayant reçu un enseignement tout au long de leur cursus scolaire sur les problèmes d'arithmétiques simples, appliquent leurs connaissances mathématiques de façon à ce qu'elles soient compatibles avec leurs connaissances sur le monde. Cet alignement sémantique guidé par nos connaissances sur le monde est observé aussi bien chez les adultes que chez les enfants (e.g., Martin et al. 2005). D'autre part, il s'avère que chez l'adulte ce processus est hautement automatisé (Bassok et al. 2008).

S'appuyer sur les connaissances familières des élèves afin de les amener à comprendre de nouveaux concepts est certainement une étape nécessaire, mais elle peut aussi s'avérer insuffisante pour les amener à une compréhension profonde et à la maîtrise des concepts enseignés à l'école. L'expérience de Moss et Case (1999) illustre bien ce phénomène et démontre comment les initiatives pédagogiques qui peuvent être menées en situation scolaire ont une influence non négligeable sur le développement des connaissances mathématiques. Dans leur étude, les auteurs ont conçu un programme destiné à des élèves canadiens de 9-10 ans scolarisés en CM1 (4th graders). Dans le but d'améliorer la compréhension et les performances des élèves dans la résolution de problèmes impliquant des nombres rationnels, le programme réorganisait l'ordre d'apprentissage des nombres rationnels. Dans ce but, et contrairement aux programmes scolaires canadiens qui préconisaient de présenter les nombres décimaux comme une autre façon de représenter les fractions, la séquence d'apprentissage consistait tout d'abord à introduire les pourcentages, 
puis les décimaux, pour finir par des séquences de mathématiques sur les fractions. Moss et Case rapportent que les enfants qui ont bénéficié de ce type d'enseignement étaient moins enclins à transférer à mauvais escient des stratégies pertinentes pour les nombres entiers mais inefficaces pour les nombres rationnels. Ainsi, ceci suggère que l'encodage des connaissances en mémoire et les liens qui peuvent être établis entre ces différentes connaissances est crucial. Ceci montre aussi comment la conception des séquences d'apprentissage peut avoir des effets sur la qualité de l'apprentissage. Prendre en compte le rôle des connaissances antérieures et familières dans la conception de programme pédagogique s'avère essentiel et indispensable. Mais ce que montrent aussi ces résultats, c'est que ces connaissances transmises et acquises à l'école peuvent avoir une incidence à plus long terme sur la compréhension scientifique des objets mathématiques.

\subsubsection{Transfert positif, négatif ou absence de transfert: quels sont les ingrédients?}

Comme nous l'avons évoqué précédemment, nos connaissances sur le monde peuvent faciliter mais aussi empêcher ou rendre difficile la découverte de solution d'un nouveau problème. En effet, dans les premières étapes de la résolution, résoudre un problème consiste très souvent à chercher à appliquer ce que l'on a appris au cours de résolutions antérieures. Cela peut conduire alors à transférer des solutions ou des stratégies acquises et maîtrisées dans des situations rencontrées précédemment qui, par analogie, sont jugées similaires à la situation nouvelle. Un autre facteur peut déterminer la nature du transfert positif, négatif ou l'absence de transfert : il s'agit de la façon dont les problèmes sont encodés et catégorisés. Le transfert sera positif quand des problèmes qui partagent le même principe de solution sont perçus comme similaires. Le transfert est négatif, quand des problèmes ne partageant pas le même principe de solution sont perçus comme similaires. Enfin, il n'y a pas de transfert quand les problèmes partageant le même principe de solution sont perçus comme dissimilaires. En d'autres termes, quand le problème source et le problème cible évoquent des structures similaires, il y a transfert. Quand, la source et la cible évoquent des structures différentes, les situations sont perçues comme différentes, les participants n'utilisant pas ce qu'ils ont appris antérieurement pour résoudre le nouveau problème. Nous développons ce point plus en détail dans la partie qui suit.

Accéder en mémoire à des connaissances transférables dans la nouvelle situation et les appliquer à bon escient déterminent le transfert réussi et la découverte de solution d'un nouveau problème. Plusieurs travaux menés dans le domaine du transfert entre problèmes isomorphes - c'est-à-dire des problèmes partageant le même principe de solution mais pas les similitudes de surface comme les personnages, les lieux, la temporalité, etc. - montrent que les difficultés rencontrées 
par le résolveur résident dans la façon dont le nouveau problème (la cible) va être interprété et catégorisé sur la base des similitudes perçues entre les situations (voir aussi les chapitres 7 et 8 dans le présent ouvrage). Ainsi, il peut arriver que les connaissances ou l'expérience passée empêchent la découverte de solution du nouveau problème : on parle alors de transfert négatif.

L'effet négatif de l'expérience passée a été décrit dès le début du $20^{\text {ème }}$ siècle par les Gestaltistes comme un phénomène de mécanisation de la pensée. Par exemple, les effets délétères de l'expérience sur la découverte de solution ont été mis en évidence dans les fameux problèmes de jarres créés par Luchins $(1939,1942)$ où il s'agit de transvaser des quantités de liquide d'une jarre à l'autre pour obtenir une nouvelle quantité. Luchins rapporte que les participants adultes qui ont résolu une première série de problèmes en appliquant une même suite de transvasements entre trois jarres, ne découvrent pas spontanément la solution de problèmes dans lesquels seul le transvasement entre deux jarres est nécessaire et suffisant. Bien que cette procédure soit plus simple que la première, la majorité des adultes continue d'appliquer la procédure connue. Dans la même veine, des études menées auprès de jeunes enfants ont montré comment la résolution d'un premier problème (problème source) pouvait avoir un effet négatif sur la résolution d'un nouveau problème (problème cible). Par exemple, Chen et Daehler (1989) rapportent les difficultés de jeunes enfants de 6 ans à ne pas appliquer de façon aveugle une solution qui leur a été enseignée précédemment dans de nouveaux problèmes qui ne partagent pas le principe de solution apprise. Les auteurs ont utilisé deux problèmes dont les principes de solution sont les suivants. Dans l'un des problèmes, la solution consiste à récupérer un objet flottant dans un récipient d'eau mais qui n'est pas accessible directement. Pour récupérer l'objet, il suffit de verser de l'eau dans le récipient afin de faire monter le niveau et attraper l'objet. La solution du second problème pour attraper un objet éloigné, non accessible directement, est de relier deux objets entre eux afin de prolonger la longueur du bras et d'attraper l'objet. On observe un transfert négatif quand les problèmes sources et cibles ne partagent pas le même principe de solution. C'est à dire que les enfants, tout comme les participants de Luchins, appliquent la procédure qui leur a été apprise lors du premier problème. Ils essaient en vain de reproduire la solution apprise et ne découvrent pas spontanément la solution au deuxième problème. En outre, il s'avère qu'entraîner les enfants à abstraire le principe de solution du problème source est bénéfique quand ce dernier partage le même principe de solution que le nouveau problème cible; mais un tel entraînement n'est pas suffisant pour amener les enfants à discriminer la non pertinence du principe de solution qui leur a été enseigné pour le nouveau problème qui ne partage pas ce principe. 
Par ailleurs, concernant l'absence de transfert entre problèmes - un constat souvent rapporté par les enseignants- il s'avère que le transfert n'est pas systématique même dans le cas où plusieurs exemples sont proposés au résolveur afin de lui permettre de catégoriser les problèmes selon leur principe de solution et ainsi de transférer la solution d'un problème à l'autre. La simple exposition et résolution de problèmes isomorphes n'est pas suffisante pour catégoriser les exemplaires comme appartenant à un même schéma de problèmes. En ce sens, des travaux rapportent que le transfert de solution entre problèmes de différents domaines n'est pas spontané si le délai entre l'apprentissage et le transfert est important ou bien si le contexte change (Spencer et Weisberg 1986; Holyoak 2005). Ce n'est qu'en incitant les participants à identifier la structure abstraite des problèmes que le transfert peut s'opérer. Par exemple, Catrambone et Holyoak (1989) ont proposé à des étudiants d'étudier, de comparer, et de résoudre des problèmes isomorphes impliquant un principe de division et de convergence des forces sur une cible à détruire (e.g., des problèmes médicaux de destruction d'une tumeur en préservant les tissus sains du patient, un problème militaire de destruction d'une forteresse en divisant par petits groupes les troupes afin d'éviter de faire exploser les routes minées qui conduisent à la forteresse). Les étudiants devaient dans un premier temps répondre à une série de questions très détaillées sur deux problèmes afin de les inciter à abstraire la structure profonde des problèmes. Puis, ils étaient invités à résoudre un troisième problème. Le principe de solution commun aux trois problèmes leur était ensuite présenté. Enfin, une semaine plus tard, les mêmes étudiants étaient invités à participer à une nouvelle expérience avant de résoudre le problème de la tumeur inspiré du problème de Dunker (1945). (voir pour une description détaillée du problème, le chapitre 8 dans le présent ouvrage). Les auteurs rapportent que plus de $80 \%$ des participants ont découvert sans aucune aide la solution qui consiste à envoyer simultanément des rayons de faible intensité convergeant pour détruire la tumeur et préserver les tissus sains. Selon Holyoak (2005), l'expertise développée par les étudiants au cours de cette expérience leur a permis d'élaborer et d'accéder en mémoire à un schéma de problèmes évoqué par un nouveau problème qui partage la même structure. On peut tirer profit de tels résultats dans le domaine des apprentissages scolaires. En ce sens, inciter les élèves à identifier, au-delà de leurs similitudes superficielles, la structure abstraite et profonde des situations est à notre sens une voie pédagogique à exploiter. C'est une des voies de stimulation de la flexibilité cognitive qui engage à changer de point de vue sur le problème et à sélectionner dans la situation les indices pertinents. En l'occurrence dans ces situations, stimuler la flexibilité cognitive consiste à entraîner les apprenants à abstraire, en deçà ou au-delà de la forme, la structure profonde du problème. 
En somme, ce qui ressort des travaux menés sur les processus à l'œuvre dans le transfert, c'est qu'un transfert efficient et flexible de connaissances acquises dans un domaine requiert d'une part une compréhension fine et profonde des situations relevant de ce domaine, mais aussi de reconnaître quand et dans quel contexte ces connaissances sont applicables. Sélectionner les indices pertinents dans la situation et identifier les contextes d'application des connaissances témoignent de flexibilité cognitive telle que nous l'envisageons. Comme le soulignent Brown et Campione :

«Un obstacle majeur à un apprentissage flexible, n'est souvent pas dû à un manque de transfert entre différents champs de connaissances mais plutôt au transfert inapproprié d'un domaine à un autre. Un apprentissage réussi implique de savoir déterminer quand, où et quoi transférer plutôt que d'appliquer aveuglément des savoirs ${ }^{1}$. » Brown et Campione 1984, p. 185

De la sorte, l'accès en mémoire et l'utilisation de connaissances qui permettent de trouver la solution d'un nouveau problème est très influencé par le contexte du nouveau problème à résoudre. C'est ce que l'on appelle les effets de contenus des problèmes et d'évocation en mémoire qui sont développés plus en détail dans les chapitres 7 et 8, respectivement, du présent ouvrage. En effet, bien plus que les similitudes de surface ou perceptives objectives partagées par les situations, le transfert entre problèmes dépend de la façon dont ces similitudes sont évoquées par le contenu des problèmes et interprétées par le résolveur (Kotovsky et Fallside 1989). Il apparaît en effet que percevoir les similitudes et les analogies est un des aspects fondamentaux de la cognition humaine. C'est en ce sens que Vosniadou et Ortony (1989) décrivent cette capacité comme cruciale dans la reconnaissance, la catégorisation, et l'apprentissage : percevoir l'analogie entre situations joue un rôle déterminant dans la découverte de solution tout comme dans le cas des découvertes scientifiques ou de la pensée créative.

Dans un domaine connexe à la résolution de problèmes, une recherche récente sur l'évocation en mémoire par analogie montre que lorsque les situations sont familières (en l'occurrence de courts scenarii décrivant des situations de la vie quotidienne), elles évoquent préférentiellement en mémoire des situations qui partagent uniquement des similitudes de structure et aucune similitude de surface. (Raynal et al. 2020). Quand les situations sont familières, alors les individus ont tendance à privilégier les similitudes de structure, et négliger les ressemblances superficielles. En revanche, quand les situations sont non familières, alors les individus auront tendance à privilégier les indices superficiels. Ce résultat conforte

${ }^{1}$ Notre traduction 
l'idée que dans le domaine scolaire, quand de nouvelles notions qui sont parfois difficiles à comprendre, entraîner les élèves à catégoriser les situations sur la base de leur structure profonde est un moyen puissant de leur permettre de construire les nouvelles connaissances sur la base d'indices pertinents.

Pour résumer, cet ensemble de recherches montrent que 1) les connaissances de l'individu, 2) son niveau d'expertise et la familiarité avec le domaine de la situation ainsi que 3) les contenus et les contextes des problèmes qui définissent les similitudes perçues entre un nouveau problème à résoudre et des problèmes résolus antérieurement peuvent contribuer à l'expression flexible des conduites de résolution de problèmes, et de façon plus générale à l'apprentissage et l'acquisition de nouvelles connaissances.

Dans cette partie nous avons centré notre propos sur le rôle joué par les connaissances évoquées par le contexte du problème et l'expertise du résolveur dans la découverte et le transfert flexible de solution. Une situation est un problème quand découvrir la solution nécessite de comprendre la structure profonde du problème (les principes de solution) et de négliger les similitudes superficielles. Dans ce contexte, la découverte de la solution qui consiste à recoder la situation, met en jeu la flexibilité cognitive qui permet d'envisager la situation d'un nouveau point de vue, de changer de représentation sur le but et de sélectionner des informations pertinentes pour l'atteinte du but (Clément, 2009). Jusqu'à présent et afin d'introduire l'objet de ce chapitre, nous avons mis la focale sur la résolution de problèmes et les processus à l'œuvre dans la découverte et le transfert de solution. Nous avons sciemment choisi d'évoquer la notion de flexibilité cognitive sous l'angle des travaux menés en psychologie cognitive dans le domaine de la résolution de problèmes comme si cette définition faisait consensus dans la littérature. Dans la section suivante, nous présenterons les différentes acceptions du terme, les nuances et les différences que l'on peut trouver selon les champs de recherche, bien que récemment on puisse établir des liens entre des approches développées dans le champ de l'Éducation, de la neuropsychologie, de la psychologie cognitive ou de la psychologie du développement (voir pour une revue de questions, Clément à paraître).

\subsubsection{Flexibilité stratégique, flexibilité représentationnelle et découverte de solution}

Le terme de flexibilité recouvre différentes significations dans la littérature scientifique sur la résolution de problèmes, notamment dans les domaines des recherches en éducation et des recherches en psychologie. En effet, certaines mettent l'accent sur la flexibilité stratégique, d'autres sur la flexibilité représentationnelle, 
d'autres encore sur le switching qui consiste à basculer son attention d'un stimulus à un autre ou d'une tâche à une autre (voir aussi le chapitre 6 dans le présent ouvrage sur la distinction que l'on peut faire entre flexibilité représentationnelle et flexibilité attentionnelle). Dans ce chapitre, notre propos porte plus particulièrement sur les recherches menées en didactique des mathématiques et en psychologie cognitive de la résolution de problèmes (pour une revue de questions sur les différentes acceptions du terme, notamment en neuropsychologie, voir Clément 2009, Clément à paraître ; voir aussi le Chapitre 2 du présent ouvrage pour une approche développementale).

\subsubsection{Les recherches menées en éducation des mathématiques}

Dans le domaine des recherches en éducation des mathématiques, la flexibilité est définie dans un sens quelque peu différent de celui défini par les chercheurs en psychologie cognitive ou en psychologie du développement. Dans ce champ de recherche, la flexibilité est généralement comprise soit comme la capacité à changer de façon flexible de stratégies afin d'arriver le plus rapidement à la solution du problème (Lemaire et Siegler 1995; Baroody et Dowker 2003 ; Kilpatrick et al. 2001 ; Verschaffel et al. 2007 ; Torbeyns et al. 2018), soit comme la capacité à choisir le format de représentation fourni par l'enseignant le plus adéquat pour résoudre le problème (Acevedo Nistal et al. 2012).

La flexibilité stratégique telle que définie par les auteurs influents en didactique des mathématiques est à comprendre dans le cadre plus général du modèle cognitif développé par Lemaire et Siegler en 1995. Selon ce modèle, quatre paramètres contribuent au développement et à l'efficience stratégique: (1) le répertoire stratégique qui correspond aux différentes stratégies dont dispose l'individu pour résoudre des problèmes; (2) la distribution des stratégies qui correspond à la fréquence avec laquelle chaque stratégie est utilisée; (3) l'efficacité stratégique définie comme la justesse et la rapidité d'exécution de la stratégie; et (4) la sélection des stratégies qui désigne le choix flexible des stratégies. Le développement des compétences stratégiques dépendraient du développement des quatre paramètres ainsi définis. En outre, selon Lemaire et Siegler, la flexibilité stratégique dépendrait de l'efficacité des compétences stratégiques de chaque individu à sélectionner la stratégie la plus rapide qui conduit à trouver la solution du problème.

Dans la lignée du modèle de Siegler, les travaux de Verschaffel et ses collègues distinguent les notions de flexibilité et d'adaptabilité qui peuvent recouvrir différentes significations selon les auteurs. Pour certains chercheurs du domaine, ces notions sont synonymes, alors que pour d'autres ces notions renvoient à deux compétences distinctes (par exemple, Heinze et al. 2009). La flexibilité stratégique décrit la capacité à choisir de façon flexible entre plusieurs stratégies disponibles en 
mémoire, mais sans nécessairement sélectionner la plus adaptée à la situation mathématique, alors que l'adaptabilité stratégique recouvre en outre la capacité à adopter la stratégie la plus adaptée au problème. Par exemple Verschaffel et ses collègues introduisent la notion de choix stratégique flexible ou adaptatif comme (Verschaffel et al. 2009, p. 343) :

«La sélection et l'utilisation consciente ou non consciente de la stratégie de résolution la plus adaptée pour un problème mathématique donné, un résolveur en particulier, et ceci dans un contexte donné ${ }^{2}$ ».

En outre, dans ce champ de recherche, de nombreux travaux ont étudié l'efficacité des différents formats des représentations externes (e.g., des graphes, des diagrammes, des tableaux, etc..) qui sont fournis aux élèves pour les aider à conceptualiser et résoudre les problèmes. Dans ce contexte, la flexibilité représentationnelle désigne la facilité avec laquelle l'élève utilise plusieurs représentions externes (Multiple External Representations) et est capable de basculer de l'une à l'autre pour résoudre le problème. Acevedo Nistal et ses collègues précisent qu'ils utilisent le terme de flexibilité représentationnelle (Acevedo Nistal et al. 2009, p. 629) :

"pour désigner les dispositions des élèves à faire le choix de représentation le plus adapté compte tenu des caractéristiques de la tâche, de l'élève et du contexte qui jouent un rôle dans la résolution des problèmes de mathématiques ${ }^{2} »$.

Dans cette perspective, la flexibilité est définie comme la capacité de l'élève à switcher entre plusieurs représentations externes et à sélectionner la représentation la plus appropriée pour résoudre la tâche. Selon ces auteurs, trois facteurs influencent cette flexibilité représentationnelle. Le premier renvoie aux caractéristiques de la tâche. L'adéquation entre les représentations externes fournies pour résoudre le problème et la solution vont faciliter l'utilisation et la sélection de la représentation la plus appropriée. Le deuxième facteur concerne les caractéristiques de l'élève. Ses connaissances conceptuelles et procédurales sur les représentations externes conventionnelles fournies en classe, ses connaissances du domaine de résolution du problème, ses préférences et habitudes d'utilisation d'une représentation externe vont déterminer la facilité d'un choix flexible entre plusieurs représentations externes. Enfin, le contexte dans lequel les tâches sont proposées détermine la flexibilité représentationnelle. Ainsi, les dispositifs fournissant un étayage actif dans la sélection des représentions externes ou stimulant la comparaison et l'évaluation

\footnotetext{
${ }^{2}$ Notre traduction
} 
des représentations externes jouent un rôle non négligeable sur la capacité des élèves à utiliser et choisir la représentation la plus pertinente.

\subsubsection{Les recherches menées en psychologie}

En psychologie, les recherches menées sur l'expression de la flexibilité en résolution de problèmes sont encore assez marginales, la flexibilité cognitive étant appréhendée dans des tâches simples de changement de règles (voir par exemple, le chapitre 1 du présent ouvrage sur les mesures de la flexibilité cognitive). Dans ce champ de recherches, la flexibilité cognitive désigne, cette fois-ci, la capacité de l'individu à changer de représentation mentale sur la situation, et non pas, comme décrit précédemment, la capacité de switcher d'un format représentationnel externe à un autre. Cette nuance est importante à souligner car souvent certains malentendus entre chercheurs de différents champs disciplinaires tiennent à la sémantique sousjacente aux concepts utilisés.

Un exemple classique de mise en défaut de la flexibilité est le phénomène de fixité fonctionnelle rapporté au début du 20ème siècle par les gestaltistes (Duncker 1945). Dunker rapporte que les participants adultes montrent de grandes difficultés à concevoir spontanément les objets dans une fonction non conventionnelle et à les utiliser de façon innovante pour résoudre des problèmes. Dans certaines situations, les connaissances sur les usages conventionnels des objets empêchent la découverte de solutions créatives. Le développement des connaissances sur l'usage conventionnel des objets dû aux expériences quotidiennes d'usage des objets familiers explique un résultat qui peut paraître à première vue paradoxal : le développement avec l'âge d'une certaine rigidité dans la découverte de solution créative. Dans une étude menée auprès d'enfants de 5 , 6 et 7 ans, German et Defeyter (2000) rapportent des effets de fixité fonctionnelle uniquement à partir de l'âge de 6 ans, les enfants de 5 ans étant beaucoup plus innovants que leurs ainés. Dans cette étude, les auteurs ont créé une version adaptée du problème de la bougie de Duncker (1945), la «Maison de Bobo l'Ours » dans laquelle on demande à l'enfant d'aider un petit ours en peluche à attraper un jouet posé hors de sa portée sur une étagère. Les enfants de l'expérience ont été répartis aléatoirement dans deux conditions expérimentales. Dans la première, une caisse remplie d'objets divers constitue une condition de pré-utilisation supposée mettre en relief la fonction usuelle de contenant de la caisse. Dans l'autre condition, les objets sont répartis autour de la caisse (condition sans pré-utilisation). La solution consiste à utiliser la caisse dans une fonction inhabituelle qui sert de marchepied pour attraper le jouet.

Alors que dans la condition sans pré-utilisation de la caisse, les enfants de 7 et 6 ans découvrent plus rapidement la solution que leurs pairs plus jeunes, dans la condition de pré-utilisation de la caisse, ces derniers découvrent beaucoup plus 
rapidement la solution que leurs ainés. Ce résultat réplique les observations rapportées par les Gestaltistes sur les effets délétères des expériences passées et des connaissances sur la découverte créative de nouvelles solutions. Toutefois, il reste à comprendre et étudier plus finement les liens entre le développement avec l'âge de la flexibilité cognitive tant dans les domaines linguistiques que non linguistiques (voir par exemple, Deák 2003, voir aussi le Chapitre 2 du présent ouvrage) et le développement d'une certaine rigidité qui apparaît au cours de l'expérience de vie. L'effet de la scolarisation et du développement d'un certain conformisme à l'adolescente pour expliquer cette rigidité du comportement est souvent évoqué. Mais, on peut invoquer aussi un principe d'économie des ressources cognitives à l'origine de tels comportements certes rigides, mais qui sont efficaces la plupart du temps dans les situations de la vie quotidienne. En effet, choisir de changer ou non de façon de faire et de penser peut s'avérer coûteux cognitivement. Néanmoins, ce constat de rigidification de la pensée liée à l'environnement scolaire et culturel incite à mener de futures recherches sur le développement de programmes pédagogiques stimulant la flexibilité cognitive pour contrer cette rigidification comportementale.

Concernant la distinction entre flexibilité stratégique et flexibilité des représentations dont nous avons parlée précédemment, nous avons pu mettre en évidence que changer de stratégie ou de procédure de résolution n'était pas suffisant pour trouver la solution à un nouveau problème qui partage les mêmes indices de surface que les problèmes résolus précédemment. C'est empiriquement ce qui a été montré dans une expérience menée auprès d'adultes à qui il était demandé de résoudre les problèmes de jarres de Luchins (Clément 2006). Dans les termes consacrés aux travaux sur le transfert, la particularité de ces problèmes, c'est qu'ils partagent les mêmes indices superficiels (le nombre de jarres disponibles), mais que le principe de solution diffère (les premiers problèmes se résolvent en utilisant les 3 jarres disponibles, les problèmes suivants en utilisant 2 ou 3 jarres, les derniers problèmes en utilisant 2 jarres parmi les 3 disponibles). Les résultats montrent que, dans le cas d'une résolution avec 2 jarres, et bien que la stratégie de transvasement soit plus simple qu'avec 3 jarres, quand ils se trouvent en impasse, c'est-à-dire quand la solution apprise avec 3 jarres ne fonctionne pas, certains participants changent de stratégie en testant de nouvelles combinaisons de transvasement tout en continuant d'utiliser 3 jarres sans découvrir la solution à deux jarres. Pour ces participants, le changement de stratégie ou de procédure peut désigner une certaine forme de flexibilité mais ne conduit pas à la découverte de solution. En effet, ces participants ne font pas preuve de flexibilité : ils ne s'engagent pas dans le traitement des propriétés pertinentes à prendre en compte, c'est-à-dire le nombre de jarres à utiliser. Ainsi, nous avons défini la notion de flexibilité représentationnelle, ou conceptuelle (voir aussi le chapitre 4 du présent ouvrage) comme la capacité à changer de point de vue, à se re-représenter le problème et le but indépendamment 
de la procédure. En d'autres termes à s'engager dans la re-catégorisation du problème sur la base du principe de solution (Clément 2009).

\subsection{Flexibilité, créativité et performances académiques}

L'intérêt pour l'étude des processus flexibles et créatifs en jeu dans les apprentissages et l'éducation n'est pas nouveau, bien que depuis les années 2000 on connaisse un développement exponentiel des travaux dans ce domaine (Craft 2005; Huang et al. 2019 ; Smith et Smith 2010, voir aussi à ce sujet la récente revue bibliométrique d'Hernández-Torrano et Ibrayeva 2020). On peut dater les premiers travaux influents sur cette question du milieu du $20^{\text {ème }}$ siècle, en référence d'une part à ceux de la Gestalt Theory dont nous avons parlés précédemment et d'autre part, à ceux de Guilford (1950) qui ont marqué le champ d'étude de la créativité. Ce dernier définissait la créativité comme une composante de l'apprentissage, et affirmait qu'une théorie de l'apprentissage complète ne saurait écarter à la fois l'insight (c'est-à-dire la découverte soudaine de la solution décrite par les Gestaltistes) et la production créative. C'est en ces termes que Guilford écrit :

"Learning theorists have had considerable difficulty with the behavior known as insight, to which creative behavior shows much apparent relationship. It is proper to say that a creative act is an instance of learning, for it represents a change in behavior that is due to stimulation and/or response. A comprehensive learning must take into account both insight and creative activity" (Guilford 1950, p. 445446).

Sans faire référence explicitement aux travaux pionniers de Guilford, il est à noter qu'aujourd'hui les politiques éducatives d'un nombre croissant de pays mettent en exergue l'importance de développer et promouvoir tout au long du cursus scolaire le potentiel créatif des étudiants (Beghetto 2010 ; Craft 2007 ; Hui et Lau 2010 ; Lin 2011 ; Mourgues et al. 2014). Toutefois, il reste encore beaucoup à faire dans ce domaine, comme en témoignent les résultats de récentes recherches qui mettent en lumière le fossé qui existe entre le potentiel créatif que des élèves expriment dans les activités extra-scolaires et le manque de créativité qu'ils peuvent exprimer dans les activités scolaires (Runco et al. 2016 ; Runco 2017).

Dans la prochaine section, avant d'exposer les résultats empiriques sur la contribution de la flexibilité et de la créativité dans les performances académiques, il paraît judicieux d'aborder les liens qui sont établis dans la littérature scientifique entre ces deux notions. 


\subsubsection{Flexibilité et créativité : quels liens ?}

Depuis les premiers travaux de Guilford (1950, 1967), le caractère flexible de la cognition a été envisagé comme une des composantes de la créativité, et son expression très variable selon les individus (Guilford 1967 ; Runco, 1995; Torrance, 1988). Selon l'auteur, la pensée créative se manifeste par deux grandes catégories d'habiletés : la première regroupe les habiletés sollicitées dans la pensée divergente, la seconde celles relevant d'habiletés de transformation.

La pensée divergente correspond à la production d'une grande variété d'idées à partir d'une situation, d'un évènement, d'un objet, etc. Selon Guilford, elle implique (1) la fluidité qui correspond à la quantité d'idées différentes produite, (2) la flexibilité, définie comme la production d'idées relevant de domaines de connaissances différents, et (3) la capacité d'élaboration de nouvelles idées. Les habiletés de transformation désignent quant à elles la capacité à produire du nouveau sur la base du connu. En termes plus actuels, ces habiletés de transformation correspondent aux processus de raisonnement par analogie que nous avons présentés précédemment. Dans ces habiletés de transformation, la flexibilité serait prédominante.

À première vue, flexibilité et créativité semblent recouvrir les mêmes processus. Toutefois, on peut distinguer deux formes de flexibilité, dont l'une est plus spécifiquement évaluée dans des épreuves de pensée divergente ou créative : il s'agit de la flexibilité spontanée. Des travaux menés en neuropsychologie auprès de patients victimes de lésions cérébrales ont montré que la flexibilité cognitive n'est pas une composante unitaire, mais peut revêtir deux formes : une forme adaptative qui consiste à s'adapter aux changements imposés par l'environnement, et une forme spontanée qui relève de la capacité à envisager un même objet ou concept sous un nouvel éclairage. En d'autres termes, la flexibilité réactive s'exprime lorsque l'environnement change et que les contraintes de la tâche exigent un changement de réponse pour une conduite adaptée. La flexibilité spontanée se manifeste quand le sujet présente des réponses variées dans un environnement stable qui ne contraint pas nécessairement au changement.

Les épreuves classiques pour évaluer la flexibilité réactive sont des épreuves de changement de règles dont une description détaillée est présentée dans le chapitre 1 du présent ouvrage. Le principe de ces épreuves est de classer des objets selon un premier critère, puis de classer ces mêmes objets selon un second critère (voir Grant et Berg 1948, pour l'épreuve originale du Wisconsin Card Sorting Test ; voir Frye et al. 1995, pour l'épreuve Dimensional Change Card Sort adaptée aux jeunes enfants). 
Les épreuves les plus utilisées pour évaluer la flexibilité spontanée sont les épreuves de pensée divergente où, comme on l'a vu, il s'agit de produire différentes solutions dans des situations-problèmes dites ouvertes, c'est à dire pour lesquelles il n'existe pas une seule solution, mais plusieurs possibles. L'une des épreuves les plus connues est l'Alternative Uses Test. Créée par Guilford en 1967, cette épreuve demande d'imaginer le plus d'usages possibles que l'on peut faire avec un objet familier, comme une brique, un parapluie ou une feuille de papier par exemple. Dans cette épreuve de pensée divergente, on mesure la fluence - c'est-à-dire le nombre d'usages différents envisagés -, l'originalité, en comparant les usages proposés par rapport aux usages fréquemment proposés, et la flexibilité qui correspond au nombre de catégories différentes (de domaines) dans lesquelles peuvent être regroupés les usages proposés.

Comme on peut le noter, les épreuves de pensée divergente sont très proches des situations de fixité fonctionnelle imaginées par les gestaltistes. Dans les deux cas, il s'agit d'imaginer des usages nouveaux d'objets familiers ; la différence repose sur le fait que dans le deuxième cas, il ne s'agit pas juste de l'évocation et de la production langagière d'usages nouveaux, mais de trouver la solution à un problème en détournant la fonction première d'un objet pour atteindre un but. Par exemple, utiliser une boite comme support plutôt qu'en tant que contenant. Ces situations de résolution de problèmes sont ainsi très intéressantes car elles sont à la fois plus proches de situations de la vie quotidienne, finalisées par un but à atteindre et impliquant la mise en œuvre par l'action de la flexibilité pour atteindre le but.

\subsubsection{Flexibilité cognitive et performances académiques}

Qu'il s'agisse des travaux scientifiques en éducation, en psychologie ou en didactique, un large consensus se dégage sur le rôle joué par la flexibilité dans les apprentissages en général, et les apprentissages scolaires en particulier. Il est à noter toutefois que les travaux portant sur les liens entre performances académiques et flexibilité cognitive sont essentiellement menés dans une conception où la flexibilité cognitive est envisagée comme un processus exécutif de basculement attentionnel shifting en anglais- d'un stimulus à un autre, ou d'une tâche à une autre. Par exemple, des travaux menés dans cette perspective montrent que les processus exécutifs - notamment la mémoire de travail, l'inhibition et le shifting- contribuent de façon non négligeable aux différences de performances scolaires des élèves (Andersson 2008 ; Best et al. 2009 ; Bull et Scerif 2001 ; Kercood et al. 2017).

Concernant plus spécifiquement la résolution de problèmes mathématiques, une question vive qui anime les chercheurs du domaine est celle de savoir si la flexibilité cognitive est une variable qui prédit les performances académiques futures des 
élèves. En effet, dans le domaine scolaire, il est communément admis que les mathématiques sont une des matières qui exigent d'être capable d'appréhender de façon flexible différents aspects des tâches et de passer de façon flexible d'une stratégie à une autre en fonction des demandes de la tâche (e.g., Agostino et al. 2010 ; Blair et al. 2008 ; Bull et al. 2001, 2008 ; Clark et al. 2010 ; Heinze et al. 2009 ; Yeniad et al. 2013). Dans une étude récente dont l'objectif était d'établir si la flexibilité cognitive prédisait les performances des élèves aux épreuves terminales d'examen de fin d'année, Hästö et al. (2019) ont proposé à des élèves finnois de classes de première $\left(11^{\text {ème }}\right.$ grade $)$ de résoudre des équations linéaires de type $(x+6)$ $+3(x+6)=21$, et de trouver des solutions innovantes au regard de la procédure classique enseignée. En effet, pour ces problèmes, la méthode enseignée dans le cursus finnois consiste en quatre étapes : 1) calculer par distribution les termes dans les parenthèses ; 2) combiner les termes ; 3) déplacer à droite les inconnues x et à gauche les autres termes ; 4) diviser par le coefficient. Cette méthode conduit aux calculs suivants en quatre étapes : $4 x+24+3 x+18=21 \Leftrightarrow 7 x+42=21 \Leftrightarrow 7 x=-$ $21 \Leftrightarrow x=-3$. Une méthode plus innovante en termes d'étapes de calcul et de difficulté à mener les différentes étapes consiste en trois étapes de résolution et conduit à calculer : $7(x+6)=21 \Leftrightarrow x+6=3 \Leftrightarrow x=-3$. Dans leur expérience, les auteurs ont mesuré la flexibilité par le nombre de solutions alternatives proposées par les élèves. Les résultats montrent que la flexibilité mesurée dans la résolution des équations est liée aux performances des élèves aux examens passés en fin d'année en mathématiques, en chimie et en langue maternelle. Toutefois, quand l'exactitude des solutions proposées est contrôlée, alors seul le niveau en mathématiques des élèves est lié à la flexibilité.

Bien que d'une façon générale les liens entre flexibilité et performances scolaires soient rapportés dans la littérature, certains travaux ne mettent pas en évidence de liens entre ces processus et les performances académiques (Espy et al. 2004; Monette et al. 2011). Comme le notent Yeniad et ses collègues, les résultats contradictoires rapportés dans la littérature sur les liens entre flexibilité et performances académiques sont sans doute liés (1) à l'hétérogénéité des tâches utilisées pour évaluer la flexibilité, (2) aux mesures pour évaluer les performances temps de réponse ou exactitude des réponses-, (3) aux caractéristiques de la population étudiée en fonction de l'âge, du sexe et du milieu socio-économique des enfants/élèves évalués, et (4) au contrôle statistique de l'impact de l'intelligence sur les performances académiques. Toutefois, les récentes méta-analyses conduites par les auteurs sur les relations entre les capacités de shifting, c'est-à-dire la flexibilité réactive,, et les performances en résolution de problèmes de mathématique et en lecture nuancent ce constat. Menant l'analyse sur 18 études concernant les liens entre les compétences en shifting et en mathématique, et 16 autres études concernant ceux entre shifting et lecture - conduites en tout auprès de 4596 sujets - les auteurs 
rapportent que les niveaux de performances les plus élevés dans les épreuves de shifting le sont aussi dans les épreuves de mathématiques. De la même façon, en lecture, les scores les plus élevés en shifting sont associés aux performances les plus élevées dans les tests de lecture.

Une interprétation possible sur ces liens entre flexibilité réactive et performances scolaires est que la flexibilité mesurée dans les recherches présentées précédemment est une compétence dont le développement est très dépendant des savoirs et expériences culturellement transmis. Les résultats rapportés récemment par Legare et al. (2018) supportent cette interprétation. Cherchant à déterminer si la flexibilité cognitive relève d'une capacité générale de contrôle exécutif, ou plutôt de savoirs et de compétences qui s'expriment différemment en fonction du type de tâche, des cultures, et des expériences éducatives, les auteurs ont proposé à des enfants d'origine américaine et sud-africaine âgés de 3 à 5 ans une épreuve de changement de règles et une épreuve d'inférence flexible de la signification de nouveaux mots (Deák et Whiseheart 2015). Les résultats montrent que dans la tâche d'inférence flexible de la signification de nouveaux mots, les enfants américains et sud-africains présentent des performances similaires. En revanche, dans la tâche de changement de règles, seuls les enfants américains scolarisés en école maternelle présentent une augmentation des performances entre 3 et 5 ans. Les auteurs concluent que le développement de la flexibilité au cours de l'âge ne serait donc pas un processus général mais dépendrait et s'exprimerait différemment en fonction de savoirs et des compétences culturellement transmis et valorisés. Selon les auteurs, l'expérience des enfants américains liée aux activités préscolaires qui impliquent de participer à des exercices fondés sur des règles arbitraires ainsi que d'interagir et de manipuler du matériel symbolique, expliquerait l'amélioration de leurs performances dans les tâches de changement de règles au cours de l'âge.

\subsubsection{Créativité et performances académiques}

Dans le domaine de l'éducation, depuis deux décennies on observe un regain d'intérêt pour l'étude de la créativité (Craft, 2005 ; Huang et al. 2019 ; Smith et Smith 2010). L'augmentation du nombre de publications scientifiques vient attester ce phénomène. Comme le soulignent Hernández-Torrano et Ibrayeva (2020), ceci est certainement dû à l'augmentation des résultats empiriques qui mettent en évidence l'implication de la créativité dans le développement cognitif et de façon plus générale qui décrivent ses bénéfices dans diverses activités de la vie sociale, affective ou professionnelle (Fanchini et al. 2019 ; Freund et Holling 2008 ; Gajda et al. 2017a ; Hansenne et Legrand 2012 ; Sternberg 2002). Cet intérêt grandissant sur le rôle joué par la créativité dans différentes sphères de l'activité humaine repose sur 
la conviction que la créativité est cruciale dans un monde de plus en plus complexe, changeant et incertain. En ce sens, les espoirs reposent sur le rôle que peut jouer l'éducation dans le développement de cette compétence (Chan et Yuen 2014 ; Davies et al. 2013 ; Scott et al. 2004).

Les recherches empiriques menées auprès de jeunes enfants montrent l'importance de la stimulation de la pensée créative pendant les premières années de la scolarité sur le développement futur de compétences transversales et transférables dans différents domaines scolaires, professionnels et personnels (Craft 2011). Le développement de pédagogies centrées sur l'apprentissage créatif tout au long du cursus scolaire et universitaire est ainsi préconisé dans plusieurs politiques éducatives européennes (Cachia et al. 2010) et états-uniennes (Adams 2005). De la sorte, de nombreux programmes d'entrainement de la créativité ont été conçus et se différencient quant aux processus cognitifs entrainés, aux techniques d'entrainement, au média support de l'entrainement, ou au type d'exercices proposés. Dans une métaanalyse conduite sur 156 programmes d'entrainement (Scott et al. 2004), il apparaît que les programmes d'entrainement basés sur les processus cognitifs impliqués dans la résolution et la formulation de problème ou la production et les combinaisons conceptuelles jouent un rôle important et bénéfique dans le développement créatif (Ward et Kolomyts 2010). Dans le même sens, des études menées auprès de jeunes enfants sur l'effet de programmes fondés sur le raisonnement par analogie montrent les bénéfices de ce type d'entrainement sur le développement cognitif d'enfants âgés de 5 à 7 ans (Antonietti 2000) et son utilité dans de nombreux domaines scolaires (Castillo 1998).

Les liens entre créativité et performances scolaires n'est pas une question nouvelle en psychologie de la créativité (Guilford 1950 ; Wallach et Kogan 1965), et les recherches empiriques menées sur cette thématique présentent des résultats ambigus, voire contradictoires. Dans une récente méta-analyse menée sur 120 recherches conduites sur les relations entre créativité et performances académiques depuis les années 60, Gajda, Beghetto et Karwowski (2017a) rapportent une relation positive, bien que modeste, entre la créativité et les performances académiques. Toutefois, cette relation positive est très dépendante des établissements scolaires où sont menées les recherches. Par exemple, dans une étude menée auprès de 589 étudiants de 34 lycées polonais, Gralewski et Karwowski (2012) montrent que le sens des corrélations entre les scores de créativité mesurés dans un test de créativité et les performances scolaires dépend des établissements où la recherche a été menée : dans certains établissements, les corrélations sont fortes et positives, dans d'autres elles sont négatives, dans d'autres encore, aucune corrélation n'a été mise en évidence. En outre, les liens entre performances scolaires, intelligence et créativité sont encore plus surprenants. Dans les établissements où la créativité a un poids 
important et est valorisée dans la réussite académique, les liens avec l'intelligence sont faibles. En revanche, dans le cas où l'intelligence est considérée comme le facteur clé de la réussite académique, le poids de la créativité est relativement faible. Les auteurs interprètent ces résultats comme la conséquence de profils d'établissements et d'enseignants différents quant aux valeurs et postures pédagogiques valorisées. En effet, les résultats suggèrent que les liens entre créativité et performances scolaires sont présents dans les classes où les enseignants soutiennent et encouragent la créativité de leurs élèves. A l'inverse, ces liens sont négatifs, ou au mieux n'existent pas, dans les classes où les enseignants ont une posture plus analytique et traditionnelle (Beghetto, 2006).

Afin de mieux comprendre les facteurs qui médiatisent l'expression de la créativité en contexte scolaire, Gajda, Beghetto et Karwowski (2017b) ont étudié la dynamique des apprentissages créatifs de 204 enfants de dix classes de l'enseignement primaire. Les auteurs ont comparé les relations enseignants-élèves au cours des discussions en classe dans trois groupes constitués ad hoc sur la base d'analyse corrélationnelle entre la créativité, mesurée dans un test standardisé (Jellen et Urban, 1986 ; Urban et Jellen, 1996), et les performances scolaires des élèves. Les élèves présentant les meilleurs scores en créativité et les meilleurs résultats scolaires (corrélation positive) forment un premier groupe, les élèves présentant les scores les meilleurs en créativité et les moins bons résultats scolaires (corrélation négative) forment le second groupe. Le troisième groupe est constitué des élèves pour lesquels aucune corrélation n'est mise en évidence entre la créativité et les performances scolaires (corrélation nulle). Les analyses conduites montrent plusieurs choses. Tout d'abord, dans le premier groupe, lors des discussions, les enseignants prodiguent à leurs élèves plus d'étayage pédagogique et émotionnel que ne le font les enseignants des deux autres groupes. En outre, la valorisation de la créativité en classe par l'enseignant est associée à un engagement actif des élèves dans les apprentissages ainsi que des prises de parole et des expressions d'idées nombreuses. Par ailleurs, dans les classes où la créativité et les performances scolaires sont positivement liées, les interactions entre l'enseignant et les élèves sont nombreuses et de qualité en laissant place aux échanges d'idées, alors que dans les classes où la créativité et les performances scolaires sont négativement liées, les interventions de l'enseignant sont plus directives, moins nombreuses et plus brèves. Enfin, dans les classes où il n'y a pas de corrélation entre créativité et performances scolaires, les questions et idées émises par les élèves ne sont pas reprises par l'enseignant, ne laissant pas place à l'exploration, au développement, et l'approfondissement des idées des élèves. Ce constat souligne l'importance, dans les programmes scolaires, d'entrainer et de valoriser le potentiel créatif des élèves. Des programmes pédagogiques centrés sur la stimulation de la flexibilité et de la créativité envisagées comme fondements des apprentissages peut être le moyen de 
répondre à cet objectif. Apprendre de façon flexible à combiner par analogie (Sander, 2000) et transformer l'existant issu des connaissances et expériences individuelles est une voie prometteuse pour qu'émergent des productions conceptuelles et/ou langagières nouvelles, originales et adaptées aux situations.

Pour résumer, l'ensemble des travaux présentés dans cette section, montrent que flexibilité cognitive, créativité et performances académiques entretiennent des relations complexes qui dépendent en grande partie des programmes pédagogiques institués à l'école, ainsi que de la posture des enseignants et des valeurs accordées à la créativité dans la réussite scolaire. Comme nous l'avons vu précédemment, la flexibilité, composante de la créativité, favorise les processus d'idéation et les habiletés de transformation. En ce sens, elle permet de réinterpréter et de réorganiser le connu afin de créer du nouveau.

\subsection{Conclusion}

Qu'elle soit envisagée comme la capacité des élèves à passer d'une stratégie de résolution à une autre et choisir la plus adaptée au problème (Lemaire et Siegler 1995 ; Baroody et Dowker 2003; Kilpatrick et al. 2001; Verschaffel et al. 2007 ; Torbeyns et al. 2018), ou bien comme la capacité à changer de point de vue sur la situation, c'est-à-dire à se re-représenter la situation et le moyen d'attendre le but en s'engageant dans le recodage sémantique du problème (Clément, 2009 ; Gamo et al. 2010 ; Gros et al. 2020 ; Gvozdic et Sander, 2020), l'ensemble des recherches menées dans le domaine de la résolution de problèmes montrent l'importance de la flexibilité cognitive dans la découverte de solutions créatives et adaptées. On a pu aussi voir que flexibilité et créativité étaient intimement liées, la flexibilité étant une des composantes nécessaires à l'expression de la pensée créative. Empiriquement démontré, le rôle crucial de la flexibilité dans les apprentissages soulève la question du défi lancé à l'école pour promouvoir une approche flexible dans l'acquisition de connaissances.

Pour conclure, les enjeux des recherches menées sur la stimulation de la flexibilité et du potentiel créatif des élèves sont à la fois des enjeux de recherche fondamentale par leur contribution au développement des connaissances sur les processus cognitifs et psychologiques impliqués dans la pensée créative, et des enjeux sociétaux et éducatifs pour "l'école de demain" confrontée à un monde où l'accès à l'information et l'innovation sont en profonde mutation. On constate d'ailleurs depuis quelques années un nombre croissant de pays où les politiques éducatives mettent en exergue l'importance de développer le potentiel créatif des élèves tout au long du cursus scolaire (Beghetto 2010 ; Craft 2007 ; Hernandez et Ibrayeva 2020 ; Hui et Lau 2010 ; Lin 2011 ; Mourgues et al. 2014). Dans un article 
consacré aux démarches éducatives innovantes menées en France, Rey et Feyfant (2012, p.2) soulignent :

"en France, l'innovation est souvent associée à l'idée d'expérimentation et vise en grande partie des ajustements locaux visant à améliorer la réussite des élèves et à personnaliser les parcours [...] sans forcément impacter directement les contenus d'enseignement. Ailleurs, l'innovation peut concerner jusqu'à l'élaboration d'un curriculum au niveau de l'école."

En d'autres termes, il paraît nécessaire et indispensable de développer des recherches dont l'objectif est d'inscrire dans les programmes scolaires français une approche éducative centrée sur la stimulation et la valorisation de la flexibilité et de la pensée créative qui ne soit pas cantonnée aux seules activités artistiques mais transversale et transférable aux différents domaines d'apprentissage, et ceci tout au long du cursus scolaire.

\subsection{Bibliographie}

Acevedo Nistal, A., Van Dooren, W., Clarebout, G., Elen, J., Verschaffel, L. (2009). Conceptualising, investigating and stimulating representational flexibility in mathematical problem solving and learning: a critical review. ZDM Mathematics Education, 41:627-636

Acevedo Nistal A.A., Van Dooren W., Verschaffel L. (2012). Flexibility in Problem Solving: Analysis and Improvement. Dans Encyclopedia of the Sciences of Learning, Seel, N.M. (dir.). Springer, Boston, MA.

Adams, K. (2005). The sources of innovation and creativity. National Center on Education and the Economy for the New Commission on the Skills of the American Workforce, Washington.

Agostino, A., Johnson, J., Pascual-Leone, J. (2010). Executive functions underlying multiplicative reasoning: Problem type matters. Journal of Experimental Child Psychology, 105(4), 286-305.

Anderson, U. (2008). Working memory as a predictor of written arithmetical skills in children: The importance of central executive functions. British Journal of Educational Psychology, 78, 181-203.

Antonietti, A. (2000). Enhancing creative analogies in primary school children. North American Journal of Psychology, 2, 75-84. 
Baroody, A. J., Dowker, A. (2003). The development of arithmetic concepts and skills: Constructing adaptive expertise. Lawrence Erlbaum Associates, Mahwah, NJ, USA.

Bassok, M., Wu, L. L., Olseth, K. L. (1995). Judging a book by its cover: Interpretative effects of content on problem-solving transfer. Memory \& Cognition, 23, 354-367.

Bassok, M., Chase, V. M., Martin, S. A. (1998). Adding apples and oranges: Alignment of semantic and formal knowledge. Cognitive Psychology, 35, 99 134.

Bassok, M., Pedigo, S. F., Oskarsson, A. T. (2008). Priming addition facts with semantic relations. Journal of Experimental Psychology: Learning, Memory, and Cognition, 34, 343-352

Beghetto, R. A. (2010). Creativity in the classroom. Dans The Cambridge handbook of creativity, Kaufman, J. C. et Sternberg, R. J. (dir.). Cambridge University Press, Cambridge.

Best, J. R., Miller, P. H., Jones, L. L. (2009). Executive functions after age 5: Changes and correlates. Developmental Review, 29(3), 180-200.

Blair, C., Knipe, H., Gamson, D. (2008). Is there a role for executive functions in the development of mathematics ability? Mind Brain and Education, 2(2), 80-89.

Brown, A.L., Campione, J.C. (1984). Three faces of transfer: Implications for early competence, individual differences and instruction. Dans Advances in developmental psychology (Vol. 3), Lamb, M., Brown, A., Rogoff, B. (dir.). Erlbaum, Hillsdale, NJ.

Bull, R., Espy, K. A., Wiebe, S. A. (2008). Short-term memory, working memory, and executive functioning in preschoolers: Longitudinal predictors of mathematical achievement at age 7 years. Developmental Neuropsychology, 33(3), 205-228.

Bull, R., Scerif, G. (2001). Executive functioning as a predictor of children's mathematical ability: Inhibition, switching, and working memory. Developmental Neuropsychology, 19, 273-293.

Cachia, Romina, R., Ferrari, A., Ala-Mutka, K. (2010). Creative learning and innovative teaching: final report on the study on creativity and innovation in education in the EU member states. Office des publications officielles des Communautés européennes (OPOCE), Luxembourg.

Castillo, L. (1998). The effect of analogy instruction on young children's metaphor comprehension. Roeper Review, 21, 27-31. 
Catrambone, R., Holyoak, K. J. (1989). Overcoming contextual limitations on problem-solving transfer. Journal of Experimental Psychology: Learning, Memory, and Cognition, 15(6), 1147-1156.

Chan, S., Yuen, M. (2014). Personal and environmental factors affecting teachers' creativity-fostering practices in Hong Kong. Thinking Skills and Creativity, 12, 69-77.

Chen, Z., Daehler, M. W. (1989). Positive and negative transfer in analogical problem solving by 6-year-old children. Cognitive Development, 4, 327-344.

Clark, C. A. C., Pritchard, V. E., Woodward, L. J. (2010). Preschool executive functioning abilities predict early mathematics achievement. Developmental Psychology, 46(5), 1176-1191

Clément, E. (2006). Approche de la flexibilité cognitive dans la problématique de la résolution de problème. L'Année Psychologique, 106, 415-434.

Clément, E. (2009). La résolution de problème. À la découverte de la flexibilité cognitive. Armand Colin, Paris.

Clément, E. (accepté, à paraître). Flexibilité cognitive et logique de découverte. Dans Les logiques de la pensée, Sander, E. (dir.). Odile Jacob, Paris.

Clément, E., Richard, J.F. (1997). Knowledge of domain effects in problem representation: The case of Tower of Hanoi isomorphs. Thinking and reasoning, 3, 133-157.

Craft, A. (2005). Creativity in schools: Tensions and dilemmas. Routledge, London.

Craft, A. (2007). Possibility thinking in the early years and primary classroom. Dans Creativity: A handbook for teachers, Tan, A. G. (dir.). World Scientific, Singapore.

Craft, A. (2011). Creativity and early years settings. Dans Developing reflective practice in the early years, Paige-Smith, A. et Craft, A. (dir.). Open University Press, Buckingham, UK.

Davies, D., Jindal-Snape, D., Collier, C., Digby, R., Hay, P., Howe, A. (2013). Creative learning environments in education: A systematic literature review. Thinking Skills and Creativity, 8, 80-91.

Deák, G. O. (2003). The development of cognitive flexibility and language abilities. Advances in Child Development and Behavior, 31, 273-328.

Duncker, K. N. (1945). On problem solving. Psychological Monographs, 58, Whole No. 270. 
Elia, I., van den Heuvel-Panhuizen, M., Kolovou, A. (2009). Exploring strategy use and strategy flexibility in non-routine problem solving by primary school high achievers in mathematics. ZDM Mathematics Education, 41, 605-618.

Espy, K. A., McDiarmid, M. M., Cwik, M. F., Stalets, M. M., Hamby, A., Senn, T. E. (2004). The contribution of executive functions to emergent mathematic skills in preschool children. Developmental Neuropsychology, 26(1), 465-486.

Fanchini, A., Jongbloed, J., Dirani, A. (2019). Examining the well-being and creativity of schoolchildren in France. Cambridge Journal of Education, 49(4), $391-416$.

Freund, P. A., Holling, H. (2008). Creativity in the classroom: A multilevel analysis investigating the impact of creativity and reasoning on GPA. Creativity Research Journal, 20, 309-318.

Frye D, Zelazo P.D, Palfai T. Theory of mind and rule-based reasoning. Cognitive Development, 10, 483-527.

Gajda, A., Karwowski, M., Beghetto, R. A. (2017a). Creativity and academic achievement: A meta-analysis. Journal of Educational Psychology, 109(2), 269.

Gajda, A., Beghetto, R. A., Karwowski, M. (2017b). Exploring creative learning in the classroom: A multi-method approach. Thinking Skills and Creativity, 24, 250-267.

Gamo, S., Sander, E., Richard, J.-F. (2010). Transfer of strategy use by semantic recoding in arithmetic problem solving. Learning and Instruction, 20(5), 400 410.

German, T. P., Defeyter, M. (2000). Immunity to 'functional fixedness' in young children. Psychonomic Bulletin and Review, 7, 707-712.

Gralewski, J., Karwowski, M. (2012). Creativity and school grades: A case from Poland. Thinking Skills and Creativity, 7, 198-208.

Grant, D. A., Berg, E. (1948). A behavioral analysis of degree of reinforcement and ease of shifting to new responses in Weigl-type card-sorting problem. Journal of Experimental Psychology, 38, 404-411.

Gros, H., Thibaut, J. P., Sander, E. (2020). Semantic Congruence in Arithmetic: A New Model for Word Problem Solving. Educational Psychologist, 55. 15326985.

Gvozdic, K., Sander, E. (2020). Learning to be an opportunistic word problem solver: Going beyond informal solving strategies. ZDM, 23(141), 1-13.

Guilford, J. P. (1950). Creativity. American Psychologist, 5,444-454. 
Guilford, J. P. (1967). Creativity: Yesterday, today, and tomorrow. Journal of Creative Behavior, 1, 3-14.

Hansenne, M., Legrand, J. (2012). Creativity, emotional intelligence, and school performance in children. International Journal of Educational Research, 53, 264-268.

Hästö, P., Palkki, R., Tuomela, D. Star, J.R. (2019). Relationship between mathematical flexibility and success in national examinations. European Journal of Science and Mathematics Education, Vol. 7, 1-13.

Heinze, A., Star, J. R., Verschaffel, L. (2009). Flexible and adaptive use of strategies and representations in mathematics education. ZDM Mathematics Education, 41, 535-540.

Hernández-Torrano, D., Ibrayeva, L. (2020). Creativity and education: A bibliometric mapping of the research literature (1975-2019). Thinking skills and Creativity, 35, 100625.

Holyoak, K. J. (2005). Analogy. Dans The Cambridge handbook of thinking and reasoning, Holyoak, K. J. et Morrison, R. G. (dir.), Cambridge University Press.

Hofstadter, D., Sander, E. (2013). L'analogie. Cour de la pensée. Odile Jacob, Paris.

Hui, A. N., Lau, S. (2010). Formulation of policy and strategy in developing creativity education in four Asian Chinese societies: A policy analysis. The Journal of Creative Behavior, 44(4), 215-235.

Huang, C., Yang, C., Wang, S., Wu, W., Su, J., Liang, C. (2019). Evolution of topics in education research: A systematic review using bibliometric analysis. Educational Review, 1-17.

Jellen, H., Urban, K. K. (1986). The TCT-DP (Test for Creative Thinking - Drawing Production): An instrument that can be applied to most age and ability groups. Creative Child and Adult Quarterly, 11, 138-155

Kercood, S., Lineweaver, T.T., Frank, C.C., Fromm, E.D. (2017). Cognitive flexibility and its relationship to academic achievement and career choice of college students with and without Attention Deficit Hyperactivity Disorder. Journal of Postsecondary Education and Disability, 30(4), 327-342.

Kilpatrick, J., Swafford, J., Findell, B. (2001). Adding it up: Helping children learn mathematics. National Academy Press, Washington, DC, USA. 
Kotovsky, K., Fallside, D. (1989). Representation and transfer in problem solving. Dans Complex information processing. What has Simon brought? $21^{\text {st }}$ symposium of the Carnegie Mellon Institute (pp. 69-108), Kotovsky, K. (dir.). Lawrence Erlbaum Associates, Hillsdale, New Jersey.

Krems, J. F. (1995). Cognitive flexibility and complex problem solving. Dans Complex problem solving: The European perspective, Frensch, P. A. et Funke, J. (dir.). Lawrence Erlbaum Associates, Hillsdale, NJ.

Lemaire, P., Siegler, R. S. (1995). Four aspects of strategic change: Contributions to children's learning of multiplication. Journal of Experimental Psychology: General, 124, 83-97.

Lin, Y. S. (2011). Fostering creativity through education: A conceptual framework of creative pedagogy. Creative Education, 2(3), 149-155.

Luchins, A. S. (1939). The Einstellung Effect in learning by repetition, Thèse de doctorat non publiée, New York University.

Luchins, A. S. (1942). Mechanization in problem solving. Psychological Monographs, 54, 248.

Martin, S. A., Bassok, M. (2005). Effects of semantic cues on mathematical modeling : Evidence from word-problem solving and equation construction tasks. Memory \& Cognition, 33(3), 471-478.

Monette, S., Bigras, M., Guay, M. C. (2011). The role of the executive functions in school achievement at the end of Grade 1. Journal of Experimental Child Psychology, 109(2), 158-173

Moss, J., Case, T. (1999). Developing children's understanding of the rational numbers: A new model and an experimental curriculum. Journal for Research in Mathematics Education, 30, 122-148.

Mourgues, C., Barbot, B., Tan, M., Grigorenko, E. L. (2014). The interaction between culture and the development of creativity. Dans The Oxford handbook of human development and culture: An interdisciplinary perspective, Jensen, L. A. (dir.). https://doi.org/10.1093/oxfordhb/9780199948550.013.16.

Novick, L. R. (1988). Analogical transfer, problem similarity, and expertise. Journal of Experimental Psychology: Learning, Memory, and Cognition, 14, 510-520.

Raynal, L., Clément, E., Sander, E. (2020). Are Superficially Similar Analogs better retrieved than Superficially Similar Analogs? Acta Psychologica, 203, 102989.

Rey, O., Feyfant, A. (2012). Vers une éducation plus innovante et créative. Dossier d'actualité Veille et Analyses, n70, 1-20, IFE, ENS-Lyon. 
Runco, M.A. (1995). Insight for creativity, expression for impact. Creativity Research Journal, 8, 377-390.

Runco, M.A. (2017). Comments on where the creativity research has been and where is it going. Special Issue: In Celebration of the Journal of Creative Behavior's 50th Anniversary in The Journal of Creative Behavior, 51(4), 308313.

Runco, M. A., Acar, S., Campbell, W. K., McCain, J., Gentile, B. (2016). Comparisons of the creative class and regional creativity with perceptions of community support and community barriers. Business Creativity and Creative Economy, 2, 24-32.

Sander, E. (2000). L'analogie, du naïf au créatif : analogie et catégorisation. L'Harmattan, Paris.

Scott, G., Leritz, L. E., Mumford, M. D. (2004). The effectiveness of creativity training: A quantitative review. Creativity Research Journal, 16(4), 361-388.

Smith, J. K., Smith, L. F. (2010). Educational creativity. Dans The Cambridge handbook of creativity, Kaufman, J. C. et. Sternberg, R. J (dir). Cambridge University Press, New York.

Spencer, R. M., Weisberg, R. W. (1986). Context-dependent effects on analogical transfer. Memory \& Cognition, 14(5), 442-449.

Sternberg, R. J. (2002). Raising the achievement of all students: Teaching for successful intelligence. Educational Psychology Review, 14, 383-393.

Torbeyns, J., Peters, G, De Smedt, B., Ghesquière, P., Verschaffel, L. (2018). Subtraction by Addition Strategy Use in Children of Varying Mathematical Achievement Level: A Choice/No-Choice Study. Journal of Numerical Cognition, 4(1), 215-234.

Torrance, E.P. (1968). A longitudinal examination of the fourth grade slump in creativity. Gifted-Child-Quartely, 12 (4), 195-199.

Urban, K., Jellen, H. (1996). Test for creative thinking - drawing production (TCTDP). Swets Test Services, Frankfurt.

Verschaffel, L., Greer, B., De Corte, E. (2007). Whole number concepts and operations. Dans Second handbook of research in mathematics teaching and learning, Lester, F. K. (dir.). Macmillan, New York, NY, USA.

Verschaffel, L., Luwel, K., Torbeyns, J., Van Dooren, W. (2009). Conceptualizing, investigating, and enhancing adaptive expertise in elementary mathematics education. European Journal of Psychology of Education, 24(3), 335-359. 
Vosniadou, S., Ortony, A. (1989). Similarity and analogical reasoning. Cambridge university Press, Cambridge.

Wallach, M. A., Kogan, N. (1965). Models of thinking in young children. Holt Rinehart and Winston Inc., New York.

Ward, T. B., Kolomyts, Y. (2010). Cognition and creativity. Dans The Cambridge handbook of creativity, Kaufman, J. C. et Sternberg, R. J. (dir.). Cambridge University Press, New York, NY.

Yeniad, N., Malda, M., Mesman, J., van Ijzendoorn, M.H., Pieper S. (2013). Shifting ability predicts math and reading performance in children: A metaanalytical study. Learning and Individual Differences, (23), 1-9.

\section{Index}

\section{A}

alignement sémantique, 4

Alternative Uses Test, 16

analogie, 5, 8

\section{C}

créativité, 14, 19

\section{$\mathbf{F}$}

flexibilité réactive, 18

flexibilité représentationnelle, 9, 10, 11,

12

flexibilité spontanée, 15, 16

flexibilité stratégique, $10,11,13$

\section{M}

mécanisation de la pensée, 6

$\mathbf{P}$

pensée créative, 15, 19, 22

problèmes isomorphes, $3,5,7$

$\mathbf{R}$

recodage sémantique, 21

\section{$\mathbf{T}$}

transfert négatif, 5, 6

transfert positif, 5 